\title{
Las consultoras de comunicación en Cataluña: 25 años de creación de espacios para el diálogo
}

\section{Communication consultancies in Catalonia: 25 years creation spaces for dialogue}

\author{
Pilar Buil Gazol. Universitat Internacional de Catalunya (pbuil@uic.es) \\ Natalia Rodríguez-Salcedo. Universidad de Navarra (nrodriguez@unav.es)
}

Recibido: 11/07/2016 - Aceptado: 15/02/2017

Resumen:

Las consultoras de comunicación comenzaron en España hace más de medio siglo. En noviembre de 1960 nació la primera agencia de relaciones públicas (RP): Sociedad Anónima Española de Relaciones Públicas (S.A.E. de RP) en Barcelona. Sin embargo, las circunstancias económicas y políticas no permitieron el pleno desarrollo de los servicios de las agencias hasta la década 1990. En los últimos años, sin embargo, el contexto de crisis económica parece haber afectado al ritmo de crecimiento. Ante esta realidad, el presente estudio aborda, tras un breve recorrido histórico por los orígenes, la evolución de las agencias de RP en Cataluña en los últimos 25 años. El objetivo reside en analizar el desarrollo de las consultoras con sede en Cataluña durante los últimos 25 años con el fin de contribuir al conocimiento sobre la historia y naturaleza de esta disciplina en el marco geográfico donde se inició. Para llevar a cabo esta investigación, se diseñó un cuestionario de diez preguntas sobre la trayectoria histórica de las agencias en Cataluña, que se envió a casi 60 empresas desde mayo de 2014 hasta mayo de 2015. Los resultados reflejan que, a pesar de la crisis, han surgido nuevas agencias. Además, se observa un desarrollo del área de las RP digitales y una preferencia por el uso del término "comunicación" en detrimento del de "relaciones públicas".

Palabras clave:

Relaciones públicas; agencias de comunicación; historia de las relaciones públicas; comunicación estratégica; Cataluña.

\section{Abstract:}

Public relations (PR) consultancies first appeared in Spain half a century ago. The first PR agency, Sociedad Anónima Española de Relaciones Públicas (S.A.E. de RP), was set up in November 1960 in Barcelona. However, due to the economic and political situation, consultancies could not fully develop the services offered until the 1990's, when they started their exponential growth in Catalonia and Spain. Over the last years, the Spanish economic crisis has disrupted the sector's rate of growth. Therefore, this study focuses on the evolution of PR agencies in Catalonia over the last 25 years in order to gain a deeper and wider knowledge of the history and nature of PR and a real picture of consultancies in Catalonia, where consultancies started half a century ago. With this aim in mind, a 10-question survey was developed and sent to almost 60 agencies from May 2014 to May 2015. Our conclusions show that new agencies have appeared in the last years and new ones are still emerging despite the crisis. Results also suggest development in new areas such as digital PR and preferences for terms such as "communication" instead of "public relations".

Keywords:

Public relations; communication consultancies; history of public relations; strategic communication; Catalonia. 


\section{Introducción}

La investigación sobre el origen y, por consiguiente, la historia de las relaciones públicas ha presentado un carácter marcadamente norteamericano hasta hace algo más de una década (Miquel-Segarra y Navarro-Beltrá, 2016; Rodríguez Salcedo, 2015; Rodríguez Salcedo, 2008). De hecho, los académicos más reputados afirmaban que las relaciones públicas modernas nacieron en Estados Unidos antes que en otra nación (Grunig, Grunig, 1992; Hiebert, 1966), al amparo del reconocimiento de la libertad de expresión, el desarrollo de los medios de comunicación y la industrialización hacia finales del siglo XIX. Durante la primera mitad del siglo XX, aparecieron los primeros consultores en relaciones públicas y se constituyeron las primeras agencias: Publicity Bureau en 1900, William Wolff Smith en 1902- o Parker \& Lee en enero de 1905-) (Cutlip, 1995).

De acuerdo con la historiografía dominante, tras la II Guerra Mundial, las empresas de relaciones públicas norteamericanas hicieron su entrada en el continente europeo y comenzaron una expansión iniciada por Hill \& Knowlton (Sotelo, 2001). Aunque el nivel de desarrollo varió entre los distintos países por razones de tipo político, económico y social, a partir de los años 60 se puede considerar que las agencias de relaciones públicas operaban ya en el continente europeo (Sotelo, 2004).

A pesar del predominio de la historiografía norteamericana (Vasquez y Taylor, 2001), en los últimos años, diversos autores han reivindicado la recuperación de otras historias nacionales para el estudio de las relaciones públicas (Bentele y Wehmeier, 2009; Bini, Fasce y Muzi Falconi, 2011; Fitch y L'Etang, 2017; Lamme y Russell, 2010; L'Etang, 2008, 2012; Miller, 2000; Raaz y Wehmeier, 2011; Rodríguez-Salcedo, 2012; Szyszka, 1997; Watson, 2014), y han logrado situar el foco en la importancia del contexto político, económico y social de un país para el desarrollo de la profesión de relaciones públicas (Rodríguez Salcedo, 2015). En ese sentido, han surgido diversos trabajos específicos sobre la evolución de esta profesión en España (Almansa, 2009; Arceo, 2004; Armendáriz, 2012; Castillo 2011; Gutiérrez y Rodríguez, 2009; Montero, et al., 2010; Noguero i Grau, 2004; Rodríguez Salcedo, 2008; Rodríguez Salcedo y Xifra 2015, Rodríguez-Salcedo, 2015; Rodríguez Salcedo y Gómez Baceiredo, 2017; Xifra 2011a y 2011b; Xifra 2012, entre otros).

Las primeras campañas de relaciones públicas en España aparecen a finales de la década de los años 50 (Armendáriz, 2012; Gutiérrez y Rodríguez, 2009; Reina, 2015; Rodríguez Salcedo, 2008; Rodríguez Salcedo, 2012; Rodríguez-Salcedo 2015; Rodríguez-Salcedo y Xifra, 2015). Si bien existe cierta controversia sobre el ejercicio de lo que hoy conocemos como relaciones públicas durante el franquismo (Sotelo, 2004; Arceo Vacas, 2006), verdaderamente fue en ese periodo cuando se constituyó la primera agencia de relaciones públicas en España y la profesión comenzó su andadura en un contexto de Planes de Estabilización para la mejora de la situación económica y de disposiciones legales como la Ley de Prensa de 1966 (Rodríguez-Salcedo, 2015). 
En noviembre de 1960 Joaquín Maestre fundó en Barcelona la Sociedad Anónima Española de Relaciones Públicas (S.A.E. de RP) (Montero, Del Río, Rodríguez, et al, 2010; Rodríguez-Salcedo, 2015). En esa misma década, aparecieron en Barcelona otras dos agencias de relaciones públicas: Jesús Ulled y Asociados, Consejeros de Relaciones Públicas S.A., que constituyó el periodista y abogado Jesús Ulled en el año 1964; y la agencia Conveplan (1963-64) (Rodríguez-Salcedo y Buil, 2015). Aunque no hay indicios documentales oficiales, la familia de Frederic Roda asegura que el escritor y crítico catalán ideó Conveplan, la tercera agencia de relaciones públicas dedicada a la promoción de la cultura, junto a Jordi Sarsanedas y José Luis Turull. En su sede comenzó a desarrollar su actividad Agustí de Uribe y Salazar, quien en 1977 abriría las puertas de su propio gabinete de relaciones públicas: Gabinete Uribe.

Al margen de estas cuatro primeras agencias catalanas, cabe mencionar otra agencia constituida en España en 1964: Jennings Public Relations, de la mano de Bernard Jennings, tal y como recoge la lista oficial de miembros de la IPRA-International Public Relations Association (1972: 51).

Poco después del nacimiento de las primeras consultoras de relaciones públicas en Cataluña, comenzaron los movimientos asociativos en busca de cierto reconocimiento para la profesión. A la Asociación Técnica de Relaciones Públicas (19611965), le sucedieron en Barcelona la Agrupación de Relaciones Públicas (1965) (De Uribe, 2015) y el Centro Español de Relaciones Públicas (1965) en Madrid. Un año después, la celebración en Barcelona de la Asamblea Anual de la International Public Relations Association (IPRA), agrupación internacional de origen europeo, propició el acercamiento y la unión de las dos asociaciones de Barcelona y Madrid en un único Centro Español de Relaciones Públicas (1966). El aparente consenso entre los capítulos de Madrid y Barcelona llegó a su fin apenas un año después, en verano de 1967, cuando las dos asociaciones volvieron a separarse.

Sin embargo, fruto de esa breve unión, la profesión contempló algunos logros como la incorporación de la asignatura "Fundamentos de psicología y teoría y técnica de la propaganda y de las relaciones públicas" al Plan De Estudios de la Escuela Oficial de Periodismo, a través de un Decreto Ministerial del 20 de abril de 1967 (Montero, Rodríguez et al., 2010; Rodríguez Salcedo, 2012; Rodríguez-Salcedo y Xifra, 2015).

El terreno abonado en la década de los 60 motivó asimismo el nacimiento de la Escuela Superior de Relaciones Públicas en 1968 en Barcelona. La creación de las Facultades de Ciencias de la Información en agosto de 1971 y el reconocimiento de las Facultades de Ciencias de la Información de Madrid, Barcelona y Navarra, abrirían las puertas a la posterior inclusión de la disciplina (1974) en los planes de estudios de estas nuevas Facultades (Arceo Vacas, 2004; Barrera, 2009; Gutiérrez y Rodríguez, 2009; Montero, Rodríguez et al., 2010). El régimen franquista se despediría además con otros logros como la Agrupación Sindical Nacional de Técnicos en Relaciones Públicas (1973) y el Registro Oficial de Técnicos en Relaciones Públicas (1975) (Montero, Rodríguez et al., 2010). 
Tras el final del régimen franquista y el establecimiento de la democracia, el siguiente hito que sin duda favoreció la consolidación profesional de esta actividad fue la llegada en los años 80 de las primeras agencias de relaciones públicas norteamericanas. La consultora Burson-Marsteller abrió sus oficinas en Madrid en 1981 de la mano de la norteamericana Teresa Dorn y posteriormente, en el año 1985, en Barcelona (Rodríguez-Salcedo y Gómez-Baceiredo, 2017). Por su parte, Hill \& Knowlton inauguró sus oficinas en Madrid en el mismo año (1981) con Nidardo Cuadrado y, en Barcelona, en 1989.

De forma progresiva surgieron diversas empresas de relaciones públicas en los años 90 (Xifra, 2011) atraídas también por el crecimiento del negocio previo a la organización de los Juegos Olímpicos de Barcelona y la Exposición Universal de Sevilla en 1992 (Llorente, 2015). Algunas procedían de capital español y, en otros casos, se trataba de nuevas agencias multinacionales que se instalaban en nuestro país. Así la función de relaciones públicas extendió sus servicios a distintos sectores. En la actualidad, diversos trabajos han abordado el desarrollo de la profesión en nuestro país y constatan su implantación en la sociedad (ADECEC, 2002; ADECEC, 2008; DIRCOM, 2000; DIRCOM, 2005; DIRCOM, 2010; PUNTO BASE, 2013; TORRES y CARRERA, 2014). La función de comunicación ha ido adquiriendo importancia en las compañías y se sigue recurriendo a agencias especializadas que se encargan de llevar a cabo (en parte o totalmente), algunos de los proyectos de comunicación (DIRCOM, 2015). Al mismo tiempo, como en cualquier otro sector, la crisis también ha afectado al desarrollo de las agencias (DIRCOM, 2014); en el conjunto de España, el sector de las relaciones públicas tuvo una caída en su facturación de más de 100 millones de euros (-23\%) en 2009. En 2010 recuperó volumen de negocio con un crecimiento del 11\% y en 2011, las cifras apenas se modificaron (2\%) (Torres y Carrera, 2015).

En este contexto, el presente artículo se centra en el estudio de la evolución histórica de las consultoras de relaciones públicas y comunicación corporativa ${ }^{1}$ en Cataluña, con sede en Barcelona, en los últimos 25 años. Se ha elegido Barcelona por estar considerada la ciudad en la que aparecieron las primeras agencias de relaciones públicas en España y porque, en la actualidad, el 27\% de las 30 agencias más importantes de relaciones públicas se encuentra en Barcelona (Torres y Carrera, 2016), una cifra que ha ido disminuyendo paulatinamente en los últimos años. En resumen, el objetivo de este artículo es ofrecer una perspectiva de la evolución histórica del sector de la consultoría en comunicación y ofrecer datos para un análisis más completo sobre esta profesión y, de ese modo, profundizar en el desarrollo de esta actividad.

\section{Metodología}

Tras realizarse una revisión de la literatura sobre el sector de las agencias, se detectó que existe una carencia de datos sobre la evolución de la consultorías en Cataluña. Por ese motivo, la investigación planteó un acercamiento al problema y se

1 Con el fin de abarcar toda la realidad también se ha incluido en la investigación a aquellas empresas que utilizan el término comunicación o comunicación corporativa. Según constataba Arceo (2004) a principios del siglo XXI, la expresión "relaciones públicas" ha perdido presencia en favor de otras denominacions como "comunicación corporativa, comunicación integral, etc., que comparten los mismos objetivos que las relaciones públicas".

40 | n² 24, pp. 37-54 | doxa.comunicación 
elaboró y envió un cuestionario a las consultoras de relaciones públicas con oficina en Barcelona en mayo de 2014. El cuestionario constaba de 10 preguntas que pretendían recopilar datos sobre la evolución histórica de las agencias o consultoras de comunicación. En concreto, se abordaron los siguientes asuntos: año de fundación, nombre originario, nombre del fundador(es), oferta de servicios en los años 80 y 90, evolución en el tamaño de la plantilla, perfiles profesionales de trabajadores, concepción de la agencia (relaciones públicas, comunicación, publicidad, etc.), procedencia del capital y contacto con multinacionales, campañas emblemáticas en los comienzos y nombre de personas relevantes en la evolución de las relaciones públicas a quienes interesaría entrevistar².

En el mes de mayo se envió el cuestionario a través del correo electrónico a 47 agencias de relaciones públicas que tenían sede en Barcelona. La relación de las consultoras se obtuvo de la información publicada en el Registro Mercantil, en las Páginas Amarillas y en diversos estudios del sector, como los informes de Torres y Carrera (2014, 2015 y 2016). Durante el mes de junio de 2014 se recogieron los resultados de la encuesta. Además, se enviaron dos mails recordatorios a las consultoras que no habían contestado para intentar recabar el mayor número posible de respuestas. Todos los mensajes recordatorios iban acompañados de una llamada telefónica para asegurar que habían recibido correctamente el cuestionario. Este proceso se alargó hasta julio de 2014.

A partir de noviembre de 2014, después de haber tenido una entrevista el 30 de octubre con el decano del Colegio de Publicidad y Relaciones Públicas de Cataluña, Ton del Pozo, se hicieron nuevos envíos del cuestionario. En concreto, se realizaron 9 envíos más a agencias de relaciones públicas y comunicación con sede en Barcelona, con el fin de alcanzar a un mayor número de consultoras.

En total, se ha contactado con 56 agencias de relaciones públicas a las que se les ha facilitado, vía correo electrónico, el cuestionario con el fin de invitarles a participar en el estudio y 29 consultoras respondieron obteniendo, por lo tanto, un índice de respuesta del $51 \%$ (Tabla I). Entras las agencias que participaron en el estudio no podría establecerse un patrón común ya que tenían características diferentes. Algunas eran agencias con más doce empleados con presencia internacional; en cambio, otras tenían un tamaño pequeño y una dimensión local; algunas eran de reciente creación y otras, en cambio, llevaban operando en el mercado más de 25 años.

En relación con las agencias que no han accedido a participar en el estudio, se debe mencionar que las razones que han apuntado han sido diversas. Así una agencia multinacional contestó: "Vi las preguntas y la mayoría de ellas no las puedo responder por temas de confidencialidad”; otra agencia multinacional con largo recorrido en el sector manifestó: “Le confirmamos que no contestamos a este tipo de encuestas. Esperamos poder serle útil en otra ocasión”; o "Después de comentarlo con nuestro DG, preferimos no participar en esta encuesta”, afirmaron desde otra consultora.

2 Ver anexo 1: tabla que incluye las 10 preguntas formuladas en el cuestionario enviado a las consultores de comunicación. 


\section{Resultados}

\subsection{Evolución de las agencias en la década de los años 90}

Algunos autores señalan la segunda mitad de la década de los 90 como el periodo de mayor crecimiento de las consultoras (Arceo, 2004; Tilson y Saura Pérez, 2003). Un estudio realizado por ADECEC señala el paso de una facturación total media de 69 millones de euros en 1996, a algo más de 150 millones de euros en 2000 (ADECEC, 2002). En efecto, los años 90 trajeron consigo un cambio estructural que hizo crecer enormemente a la industria de las PR por encima de otras del mundo de la comunicación (Álvarez, 1991).

Así las agencias de capital español que se habían constituido a finales del periodo anterior, experimentaron durante estos años un gran desarrollo. Es el caso de Inforpress (desde febrero de 2015, Atrevia) que había iniciado su actividad en 1988 con oficinas en Barcelona y en Madrid. Según los datos recogidos en el cuestionario, la empresa fundada por Núria Vilanova comenzó ofreciendo servicios de gabinete de prensa y relaciones públicas. Dos campañas emblemáticas que llevaron a cabo esa década fueron la Feria Informat y el centenario de la aspirina. Además en los 90 se crearon otras firmas en Barcelona como Bassat Ogilvy Comunicación, Solsona \& Filloy Comunicación Integral, Clotet, Presston Comunicación Internacional o Llorente y Cuenca.

Luis Bassat, el primero en inaugurar la década, funda Bassat Ogilvy Comunicación, descrita entonces como agencia de RP y de comunicación con "servicios plenos de RP". La agencia tuvo sedes en Barcelona, Madrid y Sevilla. El número de empleados con perfil de consultores osciló entre 5-55. En esos años implementaron campañas singulares, como las ceremonias de inauguración de la Expo o los Juegos Olímpicos. Cuando se constituyó era una empresa de capital español, en la actualidad se trata de una multinacional.

En noviembre de 1993 Montse Solsona y Antonio H. Filloy fundaron Solsona y Filloy, Comunicación Integral. Se trataba de una agencia con experiencia en eventos y protocolo. En 1998 la agencia cambió su denominación a Montse Solsona Comunicación, quedando Solsona como socia principal. De tamaño mediano, la agencia siempre ha contado con una plantilla entre 10 y 15 profesionales, llegando a alcanzar puntualmente la cifra de 20 empleados, entre Barcelona y Madrid, en los años más intensos. Los perfiles de los trabajadores han sido diversos, con procedencias de las Ciencias de la Información, Empresariales, Políticas y Humanidades. La presentación de los espacios públicos del Port Vell de Barcelona o la constitución del premio a la mejor secretaria del año para Manpower, fueron algunos de los proyectos en los que trabajaron y que han querido destacar en el cuestionario.

En 1995, se constituye Clotet, una consultora especializada en estrategias de comunicación corporativa y relaciones institucionales. En 1996, Xavier Roca inicia en Barcelona la agencia Presston Comunicación Internacional. La agencia, de capital catalán, se describía entonces como "una agencia de comunicación". Contaba con una plantilla constituida por 
periodistas, directores de arte y personas dedicadas a la producción, edición y maquetación, y que ha variado entre 6-16 trabajadores. En el cuestionario, señalan la macroproducción de la Opera Aida, “Tibidabo més a prop teu” y la imagen web de Planeta de Agostini como algunas de las campañas que llevaron a cabo en esta década.

En 1998, la agencia Llorente y Cuenca abrió su oficina en Barcelona. José Antonio Llorente y Olga Cuenca habían fundado esta empresa en Madrid en 1995 tras haberse formado en Burson-Marsteller Madrid. La empresa tuvo un rápido crecimiento en España y antes del año 2000 inició su expansión en Latinoamérica. En concreto, en el año 1998 abrieron su primera oficina en Lima (Perú) y en 1999, en Buenos Aires (Argentina), donde en la actualidad se han convertido en la principal consultoría de comunicación del país y la que mayor crecimiento ha experimentado en el mercado local durante los últimos años (Llorente, 2015). De hecho, en los últimos rankings de agencias de relaciones públicas mundiales aparece como la primera agencia española, en el puesto 55 de los 250 (http://worldreport.holmesreport.com/top-250). La campaña diseñada para el lanzamiento en vídeo y DVD de la película “Barbie Princesa Rapunzel” de Universal Pictures, es una muestra del trabajo que realizaban en ese periodo y que han querido destacar.

Otro hecho de este periodo que reflejan los cuestionarios es la entrada de agencias multinacionales en nuestro país. Es el caso de la agencia Weber Shandwick y Evercom, por ejemplo. En 1990, Weber Shandwick se implantó en España tras adquirir la pionera SAE de RP. Por su parte, Evercom abrió su oficina en Barcelona, como compañía independiente especializada en la consultoría estratégica y de relaciones públicas, desarrollando diversos proyectos para empresas, instituciones y administración pública en esa época.

El surgimiento de empresas de relaciones públicas especializadas merece una mención especial. Surgieron agencias de capital nacional y tamaño mediano especializadas como Miguel Ramos (1987) fundada por Miguel Ramos Tornadijo que desempeñó labores de clipping y no amplió su oferta en relaciones con los medios hasta 1992. El perfil de los profesionales que trabajan allí responde al de periodista y "en los mejores años de facturación su número osciló entre 10-15 empleados”. En 1992, Pablo Herrera Fontanals, Toni Caubet, Marcelo Ruz y Jordi Petit fundaron en Barcelona una nueva agencia especializada en publicaciones corporativas: BPMO Edigrupo. Su trabajo consistía en ofrecer servicios de diseño, redacción y gestión de publicaciones corporativas. En 10 años, crecieron de 4 a 40 empleados, lo cual refleja la demanda que existía hacia este tipo de servicios; de hecho, cinco años después, en 1997, la agencia abrió su oficina en Madrid. Posteriormente cambió su denominación a DCorporateCom, aunque su capital siempre ha permanecido totalmente español.

La agencia Nal3 Team que fundaron en 1998 Marta y Daniel Liñán en Cataluña, como agencia de relaciones con los medios, constituye otro ejemplo de agencia especializada. En la práctica, presta servicios de relaciones con los medios, eventos y patrocinio. El número de empleados osciló entre 2-11 y, según indican en el cuestionario, se trataba de perfiles variados procedentes de Económicas, Humanidades, Ingenierías o el sector de la Traducción y la Interpretación. Posteriormente modificó su nombre y pasó a llamarse Nal3 Comunicació. 
Los resultados muestran una consolidación de las agencias de relaciones públicas y de los servicios ofrecidos a finales de los años 90, así como una profesionalización en sus actividades.

\subsection{El desarrollo de las agencias en el siglo XXI}

En los primeros años del siglo XXI continúa el crecimiento de las agencias de relaciones públicas (ADECEC, 2008). Según los responsables de Nal3 Comunicación, la agencia tuvo un crecimiento sostenido del 6\% anual hasta el año 2008. Este hecho explica la aparición de agencias y la consolidación de otras como es el caso de la agencia Cincuenta y Cinco de Luis Valls (1989), dedicada a la organización de eventos, creatividad y diseño gráfico, gabinete de prensa y celebrity connection. Esta agencia que siempre ha sido de capital catalán ha contado con perfiles profesionales variados dentro de su plantilla.

Efectivamente, siguen fundándose nuevas consultoras, tales como Strategycom, iniciada en 2000 por Joan López Alegre, dedicada principalmente a las relaciones con los medios. Ha pasado de ser una agencia de prensa a una agencia integral con servicios de comunicación para pymes, instituciones y multinacionales españolas con departamentos de marketing y comunicación muy pequeños o sin ellos. Según explica su fundador, "inicialmente la empresa estaba solo integrada por periodistas y en la actualidad incluye otros perfiles como el de diseñador gráfico, experto en comunicación digital o en programación informática, entre otros. La plantilla alcanzó los 27 trabajadores en 2008 dado que también incluía la representación en Cataluña del Grupo Negocios, editor de La Gaceta de los Negocios y la revista Dinero, así como el gabinete de comunicación de AENA en Cataluña. Con la crisis, la empresa dedicada a temas publicitarios se cerró y la plantilla actual entre Madrid y Barcelona, cuenta con 10 personas. La representación de medios en Cataluña se ha retomado a finales de 2014 incorporando la programación y gestión comercial de Capital Radio en Cataluña”. En el año 2005, abrió sede en Madrid.

En 2003 Carme Miró, Kirsty Brown y Olivia Walsh constituyen Apple Tree Communications, con oficinas en Londres y en Madrid. Un año más tarde, Ramon Cobos, Alejandro Luque y Alfonso Vericat fundaron Kerygma Communication. En sus inicios la empresa tenía un carácter de consultoría aunque ha evolucionado hasta incluir: estrategia, planificación de medios, creatividad, producción (gráfica, online y audiovisual), así como posicionamiento en social media. En el cuestionario la agencia explica que este enfoque garantiza la unidad de mensaje en cada campaña y que el tono humano de todo el equipo que interviene lleve el sello de Kerygma. En la actualidad cuenta con 20 empleados entre Madrid y Barcelona. Alguno de los proyectos que ha llevado a cabo ha sido el lanzamiento del Combo de la ONCE o el diseño de la comunicación en las oficinas bancarias actuales de La Caixa y de BBVA.

En 2006, Álvaro Montoliu constituye AMT Comunicación como una agencia de comunicación. Su tamaño ha variado en función de las necesidades y los clientes que ha tenido la agencia. En la última década, la plantilla media ha sido de 4 per-

44 | n²4, pp. 37-54 | doxa.comunicación 
sonas y el capital, español. El cliente más emblemático de la agencia ha sido Singapore Airlines: la llegada y el establecimiento de su hub en el aeropuerto del Prat como primera compañía transcontinental que apostó por Barcelona frente a Madrid.

Ese mismo año, Sofía Gutiérrez de Escofet abre Soges Comunicación como una agencia que ofrece "un servicio completo de comunicación”. Los profesionales que trabajan proceden del periodismo, las relaciones públicas y la publicidad. El número de empleados ha variado entre 3-14, y la empresa es de capital catalán.

También en 2006 la multinacional Kreab Garvin Anderson inicia sus operaciones en Barcelona. Su oferta se orienta hacia la comunicación financiera, corporativa, los social media y la gestión de asuntos públicos (public affairs). El número de empleados en España se ha incrementado desde las 10 personas hasta alcanzar las 50 actuales. La agencia Alicia Comunicación abre también sus oficinas en Barcelona en el año 2006.

En 2007, Deborah Gray funda Canela Public Relations (PR). Canela PR ha ido aumentando progresivamente su plantilla según su cartera de clientes. En cambio, los perfiles profesionales, al ser una agencia joven, no han variado mucho: empleados formados en el campo de la comunicación, ya sean periodistas o relaciones públicas. Al mismo tiempo, en el cuestionario subrayan que "desde hace unos años, con el auge de Internet y el dominio de las redes sociales, todos los empleados se han ido formando en esta rama para poder ofrecer un servicio de comunicación integral a los clientes”. Canela PR siempre ha mantenido su filosofía de ser una agencia independiente de relaciones públicas. El capital de la agencia es particular de la fundadora y los socios. Sin embargo, para poder ofrecer a sus clientes servicios en otros países, forma parte de redes internacionales de agencias como Ecco PR Network ${ }^{3}$.

Durante estos años, continúan produciéndose fusiones y adquisiciones como había ocurrido anteriormente. Así la agencia fundada por José Luis Sanchís, una de las pioneras en España -comenzó su actividad en Madrid en los 70 y en Barcelona en los años 90-, pasa a formar parte del grupo internacional Incepta en 2005 y más tarde, a su vez, es adquirida por Huntsworth: cuarto grupo mundial de comunicación y relaciones públicas. Por su parte, la agencia Clotet, que se había creado en Barcelona en 1995, pasó también a formar parte de la red internacional de Ketchum, en 2010.

No obstante, según se menciona en los cuestionarios, algunas de las ofertas de adquisición provinieron de empresas nacionales y no todos los intentos de expansión cuajaron. Así, la agencia Román y Asociados, que había fundado Santiago Román en 1989 en Barcelona, tuvo varios contactos con multinacionales y empresas nacionales, pero "no prosperaron" y continúa como empresa de capital catalán. Lo mismo sucedió a Nal3Comunicació, que recibió ofertas de compra y que

Ecco International Communications Network es una red de agencias internacionales con 32 oficinas repartidas en distintos países como son Brasil, Chile, Argentina, Estados Unidos, México, Sudáfrica, India, China, Australia, Rusia, Finlandia, Polonia, Hungría, Ucrania, Irlanda, Francia, Bélgica, entre otros (http://www.ecco-network.com/members/). 
sigue conservando su capital catalán; también Cincuenta y Cinco recibió dos ofertas de compra; la empresa BPMO Edigrup mantuvo conversaciones que no se consolidaron; o Inforpress -actualmente Atrevia-que también desestimó ofertas y permanece como empresa familiar.

Otro hecho que, según los resultados, se produce en este periodo es que algunas consultoras que estaban instaladas en Madrid abrieron su oficina en Barcelona. Es el caso de ACH Consultores de Comunicación, que inaugura sus oficinas en Barcelona en el año 2010. A su vez, algunas agencias de Barcelona inician su actividad en Madrid: Canela Public Relations, en 2007.

Asimismo, continúa el proceso de expansión por parte de las agencias españolas hacia el mercado latinoamericano. Es el caso de Inforpress -actualmente Atrevia- que en 2010 abre su oficina en Brasil y a partir de ese momento, se instala en otros países del mercado latinoamericano como Colombia, Perú o Chile (Llorente, 2015). Por su parte, Llorente y Cuenca, que ya había iniciado este proceso de expansión en Latinoamérica a finales del siglo XX, continúa abriendo oficinas en esta región: Bogotá en 2000, cuya actividad se ha centrados en "proyectos de comunicación corporativa, financiera, asuntos públicos y estrategias para el sector minero energético"; Panamá en 2002; Quito en 2003; Río de Janeiro, 2008 y São Paulo en 2013; México DF, 2008; Santo Domingo, 2012 y, finalmente, en Santiago de Chile en 2014, fruto de la integración con la consultoría chilena CR Comunicaciones.

Por otra parte, la crisis económica que se inició en el año 2007 conllevó importantes cambios en el sector de las relaciones públicas. Para valorar la influencia que la crisis económica tuvo en las agencias, ayuda tener en cuenta el impacto de la recesión económica en la planificación anual y en el quehacer diario de sus clientes -los departamentos de comunicación de las compañías- (Anuario de la comunicación DIRCOM 2014). Las agencias de relaciones públicas y comunicación perdieron clientes, dejaron de recibir algunas solicitudes de colaboración y contaron con presupuestos llamativamente menores que en la década anterior. De hecho, aún no se han logrado cifras de crecimiento mantenidas (Puesto Base, 2013; Torres y Carrera, 201, 2015 y 2016).

A pesar de la merma en el volumen de negocio, han continuado apareciendo nuevas agencias de relaciones públicas en Barcelona, según muestran las respuestas obtenidas en los cuestionarios. En 2009, aparece Gotoclient como "agencia de promociones y servicios $360^{\circ}$ ", de capital catalán. Jordi Marca constituyó la agencia y en la actualidad cuenta con 7 profesionales del área de las relaciones públicas y la economía. Ton del Pozo y Joan Fondevilla fundan ese mismo año la empresa Punta Alta Comunicación, como una agencia de comunicación de capital catalán. Desde sus inicios hasta la actualidad, ha pasado de 6 a 14 empleados. Una de las campañas en que han trabajado ha sido la de concienciar a la población española del cuidado de la salud cardiovascular.

Además, en este contexto de crisis económica, las grandes agencias que sufrieron reestructuraciones, provocaron la salida de directivos que, con una dilatada trayectoria profesional, optaron por fundar sus propias agencias. Tal es el caso de Onza 
Comunicación y Marketing que nace en 2013, como "agencia de planes de comunicación y promoción”. Entre sus socios fundadores se encuentra Marta Salvat, anterior directora general de DEC (BBDO). Según comenta Marta Salvat en el cuestionario, se trata de consultores multidisciplinares capaces de definir la estrategia de comunicación de una compañía o institución al mismo tiempo que pueden asegurar su implementación.

En 2013, otras dos profesionales con una extensa experiencia en Bassat Ogilvy Communicación, Cristina de Diego y Lorena Clapers, constituyen Clapers de Diego Comunicación. Según afirman en el cuestionario, inician este proyecto "con la ilusión de ser una agencia de comunicación nacional e internacional”. En la actualidad, ocho personas integran un equipo que ofrece estrategias de comunicación y relaciones públicas, gabinete de prensa, eventos, comunicación digital y creatividad.

En definitiva, la crisis económica favoreció que aparecieran consultoras en comunicación de menor tamaño, muy competitivas, y con la visión y nivel de conocimiento de las grandes firmas. De hecho, cuando se analiza de manera global la caída del volumen de facturación, "en concreto, de los 129 millones de 2011 se pasa a los cerca de 126 en 2012, lo que supone una caída del 3\%” (Torres y Carrera, 2014), se menciona en el estudio la posibilidad de que ese descenso se deba "al traspaso de facturación de estas grandes compañías a otras firmas más pequeñas (en muchos casos microempresas) que representan en torno al 70\% de la facturación de las RRPP en nuestro país" y no tanto a una nueva caída sectorial.

En el mercado, por lo tanto, siguen conviviendo las agencias de relaciones públicas grandes con un grupo heterogéneo y numeroso de consultoras de menor tamaño, de más o menos reciente creación. Se califica como grupo heterogéneo porque existen notables diferencias desde el punto de vista del tamaño, oferta de servicios, tipología de clientes, volumen de facturación, propiedad, evolución y titularidad (Matilla, 2007). Según un estudio de ADECEC (2008), las consultoras de relaciones públicas que operan en nuestro país y que tienen capital extranjero sólo forman el 12,5\%, lo cual denota que hay muchas agencias de capital nacional. En estos momentos, de las empresas dedicadas a la consultoría en relaciones públicas, 30 están asociadas a ADECEC ${ }^{4}$, de las cuales 15 tienen oficina en Barcelona: ACH, Alicia Comunicación, Apple Tree Communication, Atrevia, Burson-Marsteller, Canela, Edelman, Evercom, Globally, Grayling, Hill \& Knowlton, Ideograma, Ogilvy Public Relations y Weber Shandwick.

En relación con el perfil profesional, en la mayor parte de las empresas de tamaño mediano con sede en Barcelona colaboran profesionales del ámbito de la comunicación que suelen ser licenciados en Periodismo, Relaciones Públicas y Publicidad, y también expertos en Finanzas o Economía.

4 Las empresas que, en julio de 2016, están asociadas a ADECEC son: ACH, Alicia Comunicación, Apple Tree Communications, Asesores de Relaciones Públicas y Comunicación, Atrevia, Berbés Asociados, Burson-Marsteller, Canela, Cohn \& Wolfe, Coonic, Edelman, Evercom, Globally, Grayling, Hill \& Knowlton, Ideograma, Ketchum, Kohl Comunicación, Lewix, LinaOrtas \& Comunicación e Imagen; Mas Consulting Group, Medialuna, Nota Bene, Ogilvy Public Relations, Planner Media, Porter Novelli, Poweraxle, Silvia Albert in Company, QUUM y Weber Shandwick. 
Al mismo tiempo, según afirman ACH Consultores en el cuestionario, el sector ha avanzado hacia perfiles más específicos, en paralelo a la propia evolución de la formación. Así, por ejemplo, el auge de las nuevas tecnologías afecta a la organización de las consultoras, siendo difícil hallar agencias que no dispongan de profesionales especializados en comunicación digital (Estanyol y Lalueza, 2013).

En las agencias de tamaño grande, como Llorente y Cuenca, los resultados reflejan un amplio espectro de perfiles profesionales procedentes de Ciencias de la Información, Administración y Dirección de Empresas, Derecho, Relaciones Internacionales, Ciencias Políticas y con postgrados especializados en Comunicación Corporativa, Asuntos Públicos y Gestión de la reputación, entre otros.

Por lo tanto, la especialización que se había dado en el periodo anterior ha continuado en los últimos años. A su vez, en la actualidad, se observa no sólo una especialización del servicio, sino también una especialización por sectores. El nacimiento de Deva Comunicación Financiera y Sostenibilidad en 2014 en Barcelona como "una agencia corporativa con foco financiero", según afirman en el cuestionario, representa un ejemplo. Esta agencia cuenta con profesionales del ámbito de la comunicación y con expertos en finanzas y sostenibilidad, una combinación que, según manifiestan, permite ofrecer a los clientes una cartera diversificada de servicios que cubre todos los aspectos de su comunicación corporativa. Gonzalo Lacalle y Juan Torres han sido los fundadores de esta agencia que es de capital español. En la actualidad cuenta además con oficina en Madrid y dispone de una plantilla fija de 13 personas más una red de colaboradores externos.

La Machi, que funda Juan Della Torre en Argentina en 2010 y llega a Barcelona en 2013, representa otro caso de consultora especializada. En sus inicios se presentó como una consultora de comunicación especializada en buenas causas y, aunque ha desechado el término consultora de comunicación por el de boutique creativa, sigue remarcando su especialización en buenas causas. "Cuando hablamos de buenas causas nos referimos tanto a instituciones que se dedican a buenas causas, como a multinacionales que quieren tener una comunicación con valores o tienen programas de RSC, etc. Ayudamos a las empresas a comunicar sus buenas causas", subrayan en el cuestionario. Amedio Comunicación es otro caso de agencia constituida en abril de 2014 por Marc Florensa y Gemma Pelegrí como agencia de comunicación especializada en prensa y 2.0.

Desde el punto de vista terminológico, como ocurría en los años 90, se observa una falta de homogeneidad en la denominación de las compañías. Los resultados muestran que la mayoría de las empresas recurren al término "agencia", "consultora" o "consultoría”. Al mismo tiempo, si bien continúa existiendo una diversidad de términos para referirse a su actividad, las empresas se inclinan más por "comunicación” que por "relaciones públicas”. Asimismo, agencias como Llorente y Cuenca subrayan la idea de dirección estratégica y gestión de la reputación. En este sentido, resulta significativo el cambio en la denominación de la agencia Inforpress (1988) en febrero de 2015 por el de Atrevia, con el fin de responder a un concepto más amplio de lo que es comunicación y no asociado exclusivamente a las relaciones con los medios. 


\section{Conclusiones}

Las tres primeras agencias de relaciones públicas en Cataluña se constituyeron en Barcelona hace ya más de medio siglo. Gracias a los cambios políticos, económicos y sociales que se iniciaron en los años 70, en las décadas posteriores se crearon nuevas agencias en Cataluña.

El sector alcanzó su más alto crecimiento en la década de los 90 y, desde entonces hasta la actualidad, las agencias de relaciones públicas y comunicación han desarrollado un papel activo en el mercado catalán. Este trabajo muestra que durante los últimos 25 años, han aparecido nuevas y variadas consultoras de comunicación y relaciones públicas -respecto a tamaño y servicios ofertados-. Al mismo tiempo, se confirma que durante los últimos ocho años, su actividad ha estado fuertemente marcada por la crisis, especialmente, por un notable descenso en el número de clientes y número empleados. Así, en la actualidad, las cifras de facturación de las agencias de relaciones públicas se encuentran alejadas de los casi 450 millones de euros facturados en 2008 en España.

Desde el punto de vista de su denominación, este trabajo constata que existe una disparidad de términos a la hora de definir la función de relaciones públicas, así como una preferencia por el término "comunicación" en lugar de "relaciones públicas". Esta conclusión coincide con los resultados de los informes anuales del European Communication Monitor de 2011 y 20155, así como con el trabajo realizado por Ruler y Verçiç (2004). Durante los últimos 25 años, las agencias han evolucionado en su denominación y han dejado el término "relaciones públicas" para optar por un concepto más amplio y estratégico de la comunicación.

En cuanto a las funciones que desempeñan las agencias y los servicios para los que son contratadas, el ámbito de relaciones con los medios, a pesar de que sigue siendo un área demandada, ha dejado de ser el único servicio o el servicio principal que ofertaban las agencias, como indica el cambio de nombre de Inforpress por Atrevia. Por consiguiente, es claro que las agencias han ampliado sus ámbitos de especialización. En este sentido, las agencias están experimentando un gran crecimiento en lo que se refiere a la comunicación digital o comunicación online, situándose en el 11,95\% de las iniciativas que las empresas desarrollan de forma externa con agencias de relaciones públicas. Asimismo se puede concluir que han aparecido agencias especializadas por sectores, como es La Machi o Deva Comunicación.

Se ha constatado que hay una diversidad de agencias en cuanto a tamaño se refiere. Cataluña cuenta con consultorías en comunicación que disponen de varios empleados en su plantilla y también con empresas unipersonales, lo cual coincide con las conclusiones de otros autores, como son Wilcox, Cameron y Xifra (2012). En este punto se confirma la importancia de las microempresas, tal como se recoge en los estudios de Torres y Carrera (2014, 2015 y 2016), así como la diversidad de perfiles que se han incorporado a este sector.

5 http://www.zerfass.de/ECM-WEBSITE/media/ECM2011-Results-ChartVersion.pdf, y http://www.zerfass.de/ECM-WEBSITE/media/ ECM2015-Results-ChartVersion.pdf 
En conclusión, se puede sostener que a principios del siglo XXI, el sector de la comunicación estratégica y las relaciones públicas se encuentra consolidado en el mercado catalán. Las agencias y consultoras de relaciones públicas instaladas en Barcelona gozan de un alto nivel de desarrollo profesional y son de capital mayoritariamente nacional. Asimismo se observa que la crisis económica no ha impedido la aparición de nuevas empresas dedicadas a las relaciones públicas y la comunicación.

Finalmente, como futura línea de investigación, se plantea la necesidad de realizar este mismo tipo de estudio en otras regiones de España con el fin de tener un conocimiento más profundo sobre la evolución de esta disciplina. Para tal fin se considera necesario superar las reticencias del sector y conseguir una mayor colaboración por parte de algunas consultoras. Este hecho, indudablemente, ha supuesto una limitación en este estudio.

\section{Referencias bibliográficas}

Adecec (2002): La comunicación y las relaciones públicas en España. Radiografía de un sector. Madrid: Pirámide.

Adecec (2008): La comunicación y las relaciones públicas en España. Radiografía de un sector. Madrid: Pirámide.

Almansa, A. (2011): Del gabinete de prensa al gabinete de comunicación: la dirección de comunicación en la actualidad.Zamora: Comunicación Social y Publicaciones.

Álvarez, T. (1991): “El sector de la comunicación corporativa inicia el despegue”, Alternativas de Marketing, 4, 60.

Armendáriz, E. (2012): “Relaciones públicas pioneras en España. Algunos precedentes en la primera mitad del siglo XX y pasos preliminares de la nueva profesión”, Anàlisi, n. 45, pp.15-31.

Arceo Vacas, A. (2004): "Public Relations in Spain: An Introduction”, Public Relations Review, n. 30 (3), pp. 293-302.

Arceo Vacas, J. L. (2006): “La investigación de relaciones públicas en España”, Anàlisi, n. 34, pp. 111-124.

Barrera, C. (2009): Historia de la Facultad de Comunicación de la Universidad de Navarra. Medio siglo de enseñanza e investigación (1958-2008). Pamplona: Eunsa.

Bentele, G. yWehmeier, S. (2009): "From Literary Bureaus to a Modern Profession: the Development and current Structure of Public Relations in Germany”, en Sriramesh, K. y Verčič, D. (eds.): The Global Public Relations Handbook. Theory, Research, and Practice. New York: Routledge, pp. 441-464.

Bini, E.; Fasce, F. y Muzi Falconi, T. (2011): “The origins and early developments of public relations in post-war Italy, 19451960", Journal of Communication Management, n. 15 (3), pp. 210-222.

Castillo, A. (2009): Relaciones Públicas: teoría e historia. Barcelona: Editorial UOC.

Cutlip, S. (1995): Public Relations History, from the 17th to the 20th century. Hillsdale: Lawrence Erlbaum Associates. 
De Uribe-Salazar, A. y Pascual, J. (2015): “El asociacionismo comunicativo en Cataluña”, en Matilla, K. (ed.): Historia de la Comunicación Corporativa en Cataluña. Barcelona: UOC, Colección Dircom, pp. 75-146.

Dircom (2000): El estado de la comunicación en España. Madrid: Dircom.

- (2005): El estado de la comunicación en España. $2^{\circ}$ estudio. Madrid: Dircom.

- (2010): El estado de la Comunicación en España. 3er estudio. Madrid: Dircom.

- (2014): Anuario de la Comunicación. Tendencias mundiales de la comunicación corporativa. Madrid: Dircom.

- (2015): El estado de la Comunicación en España. $4^{\circ}$ estudio. Madrid: Dircom.

Estanyol Casals, E. y Lalueza Bosch F. (20014): “ $¿$ Tamaño o flexibilidad? Estructura organizativa de las consultoras de relaciones públicas en España”, Sphera Publica, nº especial, pp. 135-162.

European Communication Monitor (2011): Empirical insights into strategic communication in Europe. [Disponible en http://www.zerfass.de/ECM-WEBSITE/media/ECM2011-Results-ChartVersion.pdf]

European Communication Monitor (2015): Excellence in strategic communication. [Disponible en http://www.zerfass.de/ ECM-WEBSITE/media/ECM2015-Results-ChartVersion.pdf]

Fitch, K. y L'Etang, J. (2017): “Other voices? The state of public relations history and historiography: Questions, challenges and limitations of 'national' histories and historiographies", Public Relations Inquiry, n. 6 (1), pp. 115-136.

Grunig, J. E. y Grunig L.A. (1992): “Models of Public Relations and Communication”, en Grunig J. E. et. al (eds.): Excellence in Public Relations and Communication management. Hillsdale: Lawrence Erlbaum Associates.

Gutiérrez García, E. y Rodríguez Salcedo, N. (2009): “50 años de Relaciones Públicas en España. De la propaganda y la publicidad, a la gestión de la reputación”, Doxa Comunicación, n. 9, pp. 13-33.

Hiebert, R. E. (1966): Courtier to the Crowd. The Story of Ivy Lee and the Development of Public Relations. Iowa. The Iowa State University Press.

Holmes Report (2015): The World PR Report. [Disponible en http://worldreport.holmesreport.com/top-250]

Lamme, M. O. y Russell, K. M. (2010): “Removing the Spin: Toward a New Theory of Public Relations History”, Journalism y Communication Monographs, n. 11 (4), pp. 281-362.

L'Etang, J. (2008): “Writing PR history: issues, methods and politics”, Journal of Communication Management, n. 12 (4), pp. 319-335.

Llorente, J. A. (2015): El Octavo Sentido. La Comunicación, factor clave de la sociedad del siglo XXI. Madrid: Edaf.

Miller, K. (2000): “US public relations history: knowledge and limitations”, en Roloff, M. E. (ed.): Communication Yearbook, 23. Thousand Oaks, California: Sage, pp. 381-420 
Montero, M.; Rodríguez, N.; Rodríguez, J. y Del Río Pérez, J. (2010): La edad de oro de la Comunicación Comercial. Historia de la Publicidad y las Re ᄀlaciones Públicas en España (1960-2000). Sevilla/Zamora: Comunicación Social Ediciones y Publicaciones.

Noguero i Grau, A. (2004): “Principales acontecimientos en la evolución de las relaciones públicas en España”, en Arceo, J. L. (coord.): Las relaciones públicas en España, Madrid: McGraw-Hill, pp. 337-359.

Puesto Base (2013): El sector de las relaciones públicas en España. Análisis económico. [Disponible en http://torresycarrera.com/files/Informes/Informe-PR-Spain-2012.pdf]

Raaz, O. y Wehmeier, S. (2011): “Unsichere PR-Geschichte: PR-Historiographien im systematischen Vergleich”, Medien y Zeit, n. 26 (1), pp. 6-15.

Reina, J. (2015): "Publicidad colectiva: algunas campañas primerizas de relaciones públicas a principios del siglo XX en España”, Sphera Publica, n. 15, pp. 153-172.

Rodríguez-Salcedo, N. (2008): “Public Relations before 'public relations' in Spain: an early history (1881-1960)”, Journal of Communication Management, n. 12 (4), pp. 279-293.

Rodríguez-Salcedo, N. (2012): “Mapping Public Relations in Europe: Writing National Histories against the US Paradigm”, Communication and Society, n. 25 (2), pp. 331-374.

Rodríguez-Salcedo, N. (2015): “Contributions to the History of Public Relations in the Midst of a Dictatorship: First Steps in the Professionalization of Public Relations in Spain (1960-1975)”, Journal of Public Relations Research, n. 27 (3), pp. 212228, DOI: 10.1080/1062726X.2015.1024248

Rodríguez-Salcedo, N. y Buil, P. (2015): “Las agencias de comunicación corporativa en Cataluña”, en Matilla, K. (ed.): Historia de la Comunicación Corporativa en Cataluña, Barcelona: UOC, Colección Dircom, pp. 205-266.

Rodríguez-Salcedo, N. y Gómez-Baceiredo, J. (2017): "A herstory of public relations: Teresa Dorn, from Scott Cutlip to Burson-Marsteller Europe (1974-1995)”, Journal of Public Relations Research, n. 29 (1), pp. 1-22.

Rodríguez-Salcedo, N. y Xifra, J. (2015): “Spain”, en WATSON, T. (Series ed.): Western European Perspectives on the Development of Public Relations: Other Voices. London: Palgrave Macmillan, pp. 123-138.

Ruler, B. Van y Verçiç, D. (eds.) (2004): Public Relations and Communication Management in Europe. A Nation-by-Nation Introduction to Public Relations Theory and Practice. Berlin: Mouton De Gruyter.

Sotelo, C. (2001): Introducción a la comunicación institucional. Barcelona: Ariel.

Sotelo, C. (2004): “Historia de la gestión de la comunicación en las organizaciones”, en Losada Díaz, J. C. (coord.): Gestión de la comunicación en las organizaciones. Barcelona: Ariel, pp. 35-56.

Szyszka, P. (1997): Auf der Suche nach Identität: Public Relations Geschichte als Theoriebaustein. Berlin: Vistas Verlag $\mathrm{GmbH}$. 
Tilson, D. J. y Saura Pérez, P. (2003): "Public Relations and the new golden age of Spain: a confluence of democracy, economic development and the media”, Public Relations Review, n. 29 (2), pp. 125-143.

Torres y Carrera (2016): Informe de relaciones públicas en España. Madrid: Torres y Carrera. [Disponible en: http://torresycarrera.com/files/Informes/Informe-PR-Spain-2016.pdf]

- (2015): Informe de relaciones públicas en España. Madrid: Torres y Carrera.

- (2014): Informe de relaciones públicas en España. Madrid: Torres y Carrera. [Disponible en: http://www.amic.media/ media/files/file_352_569.pdf]

Vasquez, G. M. y Taylor, M. (2001): “Public Relations: An Emerging Social Science Enters the New Millennium”, en International Communication Association: Communication Yearbook 24. Thousand Oaks: Sage Publications.

Verçiç, D. (2000): “The European public relations body of knowledge”, Journal of Communication Management, n. 4 (4), pp. 341-354.

Watson, T. (2014): Asian Perspectives on the development of public relations: Other Voices, en Watson, T. (Series ed.), National Perspectives on the development of public relations. London: Palgrave MacMillan.

Wilcox, D. L.; Cameron, G. T. y Xifra, J. (2012): Relaciones públicas: estrategias y tácticas. Madrid: Pearson.

Xifra, J. (2011a): Manual de Relaciones Públicas e institucionales. Madrid: Tecnos.

Xifra, J. (2011b): “The influence of democracy in the practice of public relations in Spain”, Anagramas, rumbos y sentidos de la comunicación [online], n. 10 (19), pp. 69-87. [Disponible en: http://revistas.udem.edu.co/index.php/anagramas/article/view/519/471]

Xifra, J. (2012): “On decrees, disputes and definitions”, Communication Director, n. 4, pp. 40-43. 
ANEXO 1. Tabla con 10 preguntas del cuestionario enviado a las agencias de comunicación

\section{PREGUNTAS CONTENIDAS EN EL CUESTIONARIO ENVIADO A AGENCIAS}

\begin{tabular}{|c|l|}
\hline 1 & ¿En qué año comenzó su actividad la agencia en España? ¿Y en Cataluña? \\
\hline 2 & ¿Qué nombre tenía la agencia? \\
\hline 3 & Nombre del fundador/fundadores. \\
\hline 4 & ¿Qué servicios ofrecía su agencia en la década de los 80 y de los 90? ¿Y a partir del año 2000? \\
\hline 5 & ¿Qué variación ha habido en el número de empleados/plantilla en el tiempo? \\
\hline 6 & ¿Qué perfiles profesionales hay en la agencia? \\
\hline 7 & ¿Cómo se describía o presentaba la agencia en sus inicios? ¿Y en la actualidad? \\
\hline 8 & $\begin{array}{l}\text { ¿Tuvieron algún contacto u oferta de compra por parte de otras multinacionales del sector? ¿Su capital ha sido } \\
\text { catalán... español... o internacional? }\end{array}$ \\
\hline 9 & $\begin{array}{l}\text { ¿Recuerda campañas emblemáticas que se hicieron desde su agencia y que constituyan una muestra del trabajo } \\
\text { que se hacía en la década de los 80 o de los 90? }\end{array}$ \\
\hline 10 & $\begin{array}{l}\text { ¿Podría facilitarnos, por favor, el nombre de otras personas a quienes convendría entrevistar con motivo de este } \\
\text { trabajo (fundadores de agencias, profesionales destacados, defensores de la profesión en sus comienzos)? }\end{array}$ \\
\hline
\end{tabular}

